\title{
Chinese Herbs
}

National Cancer Institute

\section{Source}

National Cancer Institute. Chinese Herbs. NCI Thesaurus. Code C2663.

Herbs used in Chinese Herbal Therapy for toxicity attenuation. (NCI) 